\title{
Research in the Use of Product Semantics to Communicate Product Design Information
}

\author{
Chung-Hung Lin \\ No.1, Lingtung Road, Department of Technological Product Design, Lingtung University, \\ Taichung, Taiwan \\ chunghung@teamail.1tu.edu.tw
}

\begin{abstract}
In the past twenty years, following the transformation of industrial technology and structure, needs created by modern life, issues regarding environment protection, and simply new ways of thinking have revolutionized the core value of product design. There is more to be considered than just the convenience that a product can bring. Consumers nowadays also want to learn how to use a new product in the most efficient way, and many industrial designers see that as one of their goals. The use of Product Semantics in product design is a proper means to communicate the information that consumers need in order to improve their overall experience using the product. Recent research focuses on the application of product design semantics, in order to discover how designers convey messages through the use of semantics, i.e. styles, colors, functions and textures, etc. It seeks to determine how the best way for the designer to communicate all that information to the consumer. A designer want the user to be able to operate his product under "zero obstacle" conditions, to understand the message the product carries, and to enhance the consumer's pleasure and comfort during its operation. From the perspective of industrial design, there are two summarized main points:
\end{abstract}

1. Product design is now defined as a system to communicate product information based mainly on product use circumstances.

2. The message that a product carries has become the most important factor throughout the design process.

Keywords: Product design, product semantics, message communication, designer.

\section{Preface}

In today's society, our living environment has become focused on "people" instead of "objects". Therefore the main focus of design is humanism, and the style of the product should be focused on communication with "people". The purpose of design is to express a designer's idea, which means giving the designed object ways to communicate feelings or meanings. In conclusion, the supreme goal of product design is for the operator to understand and complete the operation. Under this principle, a product carries a mission of "translation" and is the bridge between a user and a designer. Therefore, product semantics can be used to discuss psychology and changes in our society or culture. The foundation of product design is based on "people", and also 
needs to include factors coming from different groups, history and cultural backgrounds. Shortening the distance between designer and consumers is the first goal of product design. Creating useful, functional products is the primary task of the modern industrial designer.

Nowadays professional designers are widely recognized for their outstanding contributions which is important in product function. On the other hand, when it comes to product design, modern society has come to understand that the focus should definitely be on people (users). The need for a human-oriented approach to product design has given rise to the use of "semantics" as a factor in good product development. One of the most widely discussed issues in product design is how to convey "the meaning" of the product. According to Dr. Krippendorff, product designers need to consider differences in perception, culture and history, along with different symbols. These topics are meant to encourage designers to focus on "people" in product design.

This also gives industrial designers another direction for product design. Since the display of a product affects a person's feelings, Product Semantics can be useful for discovering consumers' affections, sentience and symbols. Furthermore, it discusses how to think to make a proper message communication while using a product [1]. While encouraging an increased psychological need for a product, in addition to its core function, the product should also communicate culture essence, meaning and circumstances of usage, and various symbols.

From the foregoing statements, one can conclude that Product Semantics is the study of existing symbols and their meaning under regular circumstances. The designer also needs to consider individual users' varying physical and physiological functions, as well as various mental, social and cultural phenomena. Recent research has sought to determine whether "Product Semantics" is a suitable criterion for product design. The answer should be positive; not a stiff rule but a flexible principle under the consideration of "humanness".

\section{Review of Literature}

\subsection{The Origin of Product Semantics}

In 1983, Prof. Klaus Krippendorf and Prof. R. Butter first launched the concept of "Product Semantics" which they defined as "Research on the symbols of usage under different styles of people's creation, and use the idea in design." In 1984 Krippendorf further defined Product Semantics as an awakening from the old way. Products, he said, should represent more than just physical functions. Products themselves should show or imply to consumers how they are to be operated. In addition, products should carry symbolic meanings and should be able to blend into people's lives.

The theory of Product Semantics comes from language symbol system. Semiotics is the practice of using symbols in scientific and phenomenological research. Saussure, the father of Semiotics, said "Language is a semiotic system to express ideas." $\mathrm{He}$ believes each symbol has at least two meanings. A symbol is a "Signifier"-a symbolic model of an object. In addition, a symbol is also "Signified", communicating the meaning behind the symbol, which can be an idea, cultural essence or other symbolism. Professor M. McCoy at the Cranbrook Academy of Art in America considers the following five features of product semantic design when evaluating product styling: 
1. Environmental Context: Forms, sizes, material and colors of a product should be in symmetry with its natural environment and social circumstances.

2. Memory: When a new product is launched, it doesn't necessarily need a brand new modeling language, but should seek to use memories people share in old images instead. Product consonance can be created by the use of familiar sequences and messages.

3. Operation: In order to properly guide consumers through standard operation of the product, operating instructions should be clear and easy to understand. They should thoroughly describe the control, display, appearance, material, and color of the product, and should explain the function and relationship of every control key, switch, and operating device.

4. Process: Semantic design is not about making products mysterious. On the contrary, the invisible inner operation needs to be proclaimed through outward display. Even though there are many things the realms of new technology that one cannot witness directly, designers need to interpret the inner operation of a product for the consumer. Not only must the designer communicate the technical function of a product, but he should also encourage the consumer to use his heart or intuition to envision its operation.

5. Ritual of Use: Simple styles can satisfy the needs of simplicity and efficiency in our daily lives. On a ceremonial occasion, for example, a product can be given an alternative design interpretation. A product can be made solemn, warm, cold or rational in order to complete a scenario design and foster the desired psychological interaction between the object and its user.

\subsection{The Definition of Product Semantics}

The origin of Product Semantics in industrial design can be traced to the 1960s. Semantics is literally the study of the meaning of words. Semantics is the science of exploring and researching meanings of languages. "Product Semantics" is a concept borrowed from linguistics; coming originally from semiotics. However there is a social, historical, and philosophical background behind its existence.

Product Semantics is the study of the images and meanings of a product. A product's style, color, texture, function or context form the meaning and concept that the product seeks to convey. Just as semantics is the study of the meaning of language in general, Product Semantics is the study of Product Language. The structure of Product Semantics theory comes originally from "the use of symbols " from Hochschule für Gestaltung, in Ulm, Germany. To trace its history back even farther, it is related to the "theory of marks" from Charles and Morris at the New Bauhaus School of Design in Chicago. The Product Semantics concept was launched in 1983 by K. Krippendorf in America and R. Butter in Germany, and was defined by Cranbrook Academy of Art in America in a seminar of Product Semantics held by IDSA as follows: "Product Semantics is research into the interaction between the operation and the message that is carried by a man-made object (Figure 1), and how we use that knowledge in industrial design .’[2] 


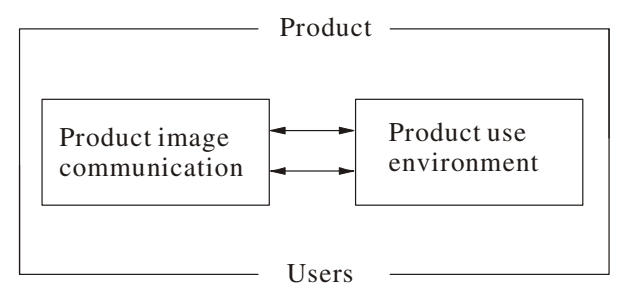

Fig. 1. The interactive relationship product messages and product use

\subsection{Marks and Symbols of Product Semantics}

Marks and symbols are signs for things, or are devices that can be used to distinguish status. There are numerous ways to show them including words, pictures, colors, images and actions, and psychological characteristics are created by images in people's minds, through a concept or impression. Signs and symbols are built through sensory perception, consciousness or knowledge, or through a sequence of psychological activities. By reflecting on an object, an idea or a thought can be associated with one of the object's features.

The theory of "Semantics" teaches us that people's knowledge or feelings are values or impressions on their minds created by sight, hearing, touching, memories or imagination which are received by them as signs and symbols in the first place. Product semantics is based on "signs". A product's appearance -its shape, color, texture, and materials-convey information to a user that enables him to see it and touch it accurately. Additionally, when it comes to the style of a product, designers should take into consideration the differences in cultures, customs or behaviors in order to find the true meaning of a sign (Figure 2).

Generally speaking, judging from inward and outward aspects of a product, there are a sequence of design considerations including functions, structures, shapes, materials, colors, interfaces, human factors... that must coalesce to form a product. The ultimate objective of a product is not only to satisfy its functional requirements, but also to take the user's psychological circumstances into account through the use of Product Semantic Signs and Symbols which communicate the meaning of the design by integrating semantic forms, colors, and textures.

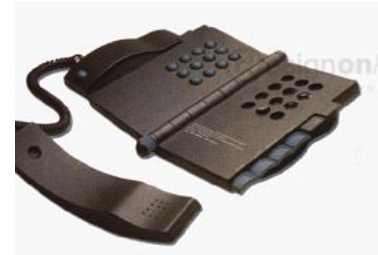

Fig. 2. The basic component of Product Semantics is "signs" 


\section{Semantic Forms}

There are two main concepts in the theory of Product Semantic Forms: One is the meaning of the form and the other is the message of the form. The analysis of the structure of semantics belongs to expressions of meanings. Messages communicated through Semantics help a designer understand the affects of a product's form on a user, and also help deliver the messages accurately. In product design, semantics is necessary for communicating symbolic messages. According to Pierce's theory, there are three circumstances in which the use of Product Semantics is appropriate as a means to convey a product's symbolic messages:

1. An accurate display of the meaning of a product can enhance the quality of the interface for a user.

2. Signs or symbols are used in communication between designers, and are a very helpful coordination element for users as well.

3. The ways to display product signs or symbols are not necessarily alike, but depend on the specific society, culture, and customs in which the product is marketed.

Product Semantics involves two main concepts: "The appearance of the meaning of a form" and "The conveyance of the message of a form." Analysis of the structure of semantics belongs to expressions of meanings. Messages should be conveyed through Semantics so the designer can understand the effects of a product's form on a user and can deliver the product's messages accurately. As illustrated in the following diagram, Product Semantics has three principal aspects, "product signs", "the appearance of a meaning" and "the conveyance of a message". (Figure 3)

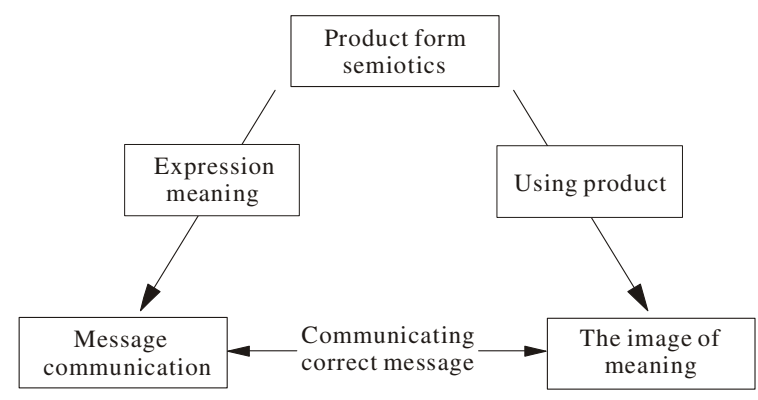

Fig. 3. Relations among signs, meanings and messages

In light of the foregoing discussion, the focus of our research is "the communication of product information". From there we extend the meaning and symbol of product forms, and are thereby able to distinguish the meaning of signs and symbols in practice. Moreover, according to the description of signs and symbols, we can also determine every model (forms, textures, lines, etc) of a sign in order to establish the existence of "Semantic Product Forms".[3] 


\section{Semantic Colors}

The expression of color is the most abstract part of Product Semantics. Color is a bridge between the meaning of a product and the consumer's emotions. People have strong, direct feelings and impressions toward colors. In product design, colors are not only beautiful and decorative, but they also carry symbolic meanings. As the primary visual factor for appreciation of beauty, colors deeply affect our visual experience and emotions. Colors play an important role in forming a conception. Colors and styles that are in symmetry in a single design bring meaning and life to a product. Semantic colors in product forms come from people's visual and physical reactions to colors. This process allows users to connect their own experience with a product, and to discover meanings that a product carries.

Colors in a product design are expressed by Hue, Value and Saturation and their relationships. Different colors and combinations can arouse different emotions: red symbolizes passion and vitality, blue represents distance, calm and tranquility, purple means mystery, white represents simplicity, black shows solemnity, nobility and old age, grey is modest, etc. Every color carries different emotions and represents different symbolic meanings.

The careful use of colors in product design can not only display the meanings of functions, and ways of operation, but can also make users feel more comfortable. For example, a red button can indicate an emergency switch, a green button generally means safe for operation, and a yellow button can be used to signal precaution. Shapes and colors of a product carry meanings as well. For example, black and silver surfaces look edgy and solemn. That is why many $3 \mathrm{C}$ products are in silver-gray or black, although some are combined with yellow, red, or green to add a sense of fashion. If products such as purses or shoes are rendered in red, pink, or other warm colors, they will carry a graceful female taste. White is a very classic color. The Macintosh computer from Apple is white, which carries a vivid emotional and physical character and has become the image for Mac. MUJI, on the other hand, is based on a simple, natural design.

\section{Semantic Materials}

Semantic Materials is a branch of Product Semantics that deals with material properties, textures and tissues... compounds that deliver messages. In addition to styles, signs or symbols, lines, etc, the meaning of a product is received through being seen and touched by a user. When designers choose materials, the first consideration is function; for example, intensity, wear resistance, and process ability, as well as possible user-defined requirements. Textures and tissues of materials give the user a visual and physical experience with a product that also generates emotions and certain symbolic meanings. Therefore, designers need to take people into consideration when choosing materials. Textures and tissues are forms of art, and the right material enhances emotional feelings and shortens the distance between products and people.

Different textures bring out different emotions. Glass and steel, for example, are edgy and modern, as in a SONY laptop computer, where the surface is made of clear compound metal to give a sense of transforming technology. By contrast, wood and bamboo, are natural, relaxing and down-to-earth. Wraps used by MUJI are in a wood pattern, giving a sense of nature and seeming more environment-friendly. Patterns 
(lines, arrangements and setups) are also signs and symbols that can help users understand the meaning of product functions. For example, several thin lines are used to prevent slipping, a rough surface is designed for holding, and a dented part invites the user to press down. Texture of materials and performance characteristics of tissues affect the ultimate visual result of a product.

\section{The Display of Signs in Semantic Product Forms}

An analysis based on Semantic theories from Peirce, Sanssure and Morris will still need to be proven in practice. It's based on a designer's understanding of Semantics that shows the meaning a product carries (Figure 4). Therefore, we need to know how to apply Semantics to explain values of styles of products, so designers should view a consumer's questions as information.

Product semantics is about communication which means every message that a product can deliver to a user.[4] The message here is an application of signs. Generally consumers express their inner thoughts into outward behaviors, then eventually an understanding. For example, the button on a toaster is often a long, vertical gap which people associate with the idea of pressing down. For example, if users can tell a button is for turning on and off from its shape, texture, color, then guide a user how to operate by its special pattern, and through signs or colors let a user make various adjustments such as turning the volume up, down or changing the . A user can get used to all of the operation through this kind of message convey. As for the product which but a symbolic meaning, it's a practical simulation, a clear display. If we apply icon, index and symbol three signs from Peirce's Semantics into product design, it will create a sequent of solution as in Figure 5.

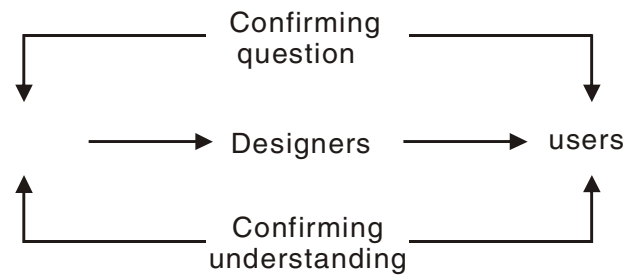

Fig. 4. The transformation of positive product meaning

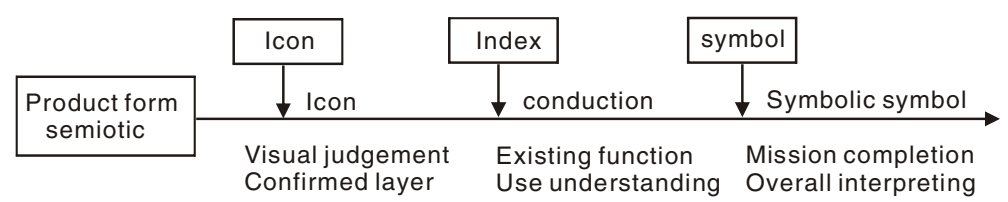

Fig. 5. Application of Perice's Semantics (adapted by the author) 
Table 1. The meaning of product semiotics (adapted by the author)

\begin{tabular}{|l|c|c|c|c|c|}
\hline Form & Meaning & Material & Meaning & Color & Meaning \\
\hline \hline Circle & Rotatable & Trace & $\begin{array}{c}\text { Preventing } \\
\text { slides }\end{array}$ & Red & Warming \\
\hline Square & $\begin{array}{c}\text { Canbe } \\
\text { pushed }\end{array}$ & Coarse & Technology & Black & High quality \\
\hline Spiral & Adjustable & Glossy & Fashion & Gray & Male \\
\hline Concavity & $\begin{array}{c}\text { Canbe } \\
\text { pressed }\end{array}$ & Tiny holes & Exhausted & Green & $\begin{array}{c}\text { Environmental } \\
\text { protection }\end{array}$ \\
\hline
\end{tabular}

Now we can find out that product signs are based on people's transition from cognitive behaviors into the message of the need of operation, the efficient convey is no more than "interpretation of meanings." As for an appearance of a product, it gives people some visual signs, theses signs are special "languages" that a product carries, and these languages affect people's opinions of the product. Symmetrical Cartesian geometries shows the solemnity of a structure and creates a peaceful, graceful and cheerful atmosphere. Solemn but lively circles have a sense of inclusion which is good for creating a satisfactory and harmonious atmosphere. Curves represent movements and vitality and can be used to create a enthusiastic, free and friendly atmosphere. Free curves are close to the nature and full of life and can be used to create a simple, natural and environment friendly atmosphere. These signs are attached to a product whether a designer put them there intentionally or not and affect a user to feel positive or negative.[5]There are three most common product signs which are shapes, textures and colors. The meanings are categorized as the following Table 1.

\section{The Study of Cases of Product Design}

The research is to study various signs and see if the messages that a product carries can guide a user to operate successfully and allow a user to achieve the goal of efficient product operation. And how do we apply Product semantics into product design to show the meaning of a product or its symbolic meaning is the key of making a product alive. Therefore, in order to show that the theory of sign and semantic product theory are helpful to making a product easier to understand and closer to consumers' needs. In the research, the concept is a flash light design along with the theory of sign and Product semantics. Case 1: The object is a tall triangular prism, there is a small cylinder and light on the top. On the edge, there is a small triangle switch facing up. This kind of design implies a force pushing towards the tip and is able to switch the light on. This design is a kind of expression of symbol as long as how we find out the meaning of an arrow (Figure 6).

Case 2: The object is a cone, the head is big and the end is small, the light bulb is at the big end, the light at the end is expending in a cone shape, and the handle is triangle which implies pressing down with a palm to turn on the light. A design like this is in a geometry form which has a convergent effect and the handle can spread up (Figure 7). 


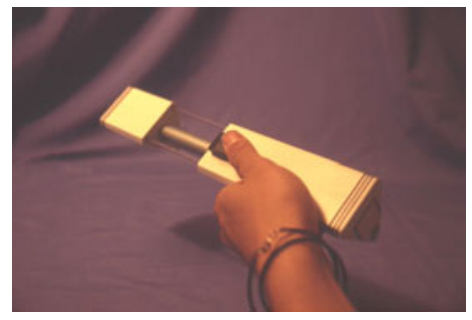

Fig. 6. Design of a triangular prism flash light

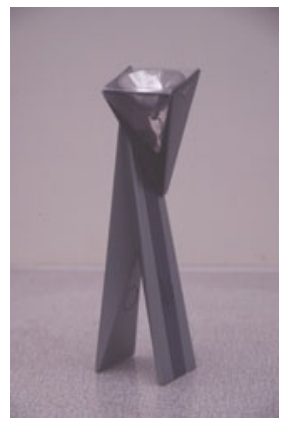

Fig. 7. Design of a corn shape flash light

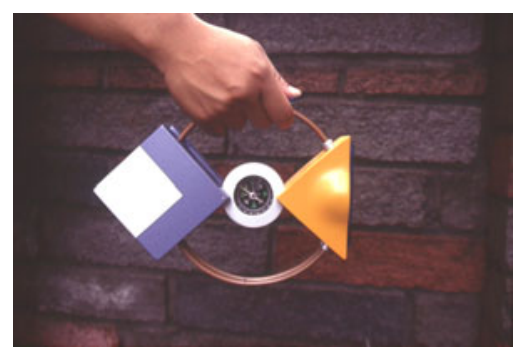

Fig. 8. Design of a triangular prism shape flash light

Case 3: In the middle place, a small circle attached to a square and a triangle on the both sides, then from the square and triangle extends a big round line, a circle means being able to spin around this small object, and also able to adjust the relative position of the square and the triangle. And about the shapes, a square means a bottom, a triangle means gathering and releasing the light, and using yellow to mark where the light is (Figure 8).

After the discussion of the three cases above, we can see whether shapes, colors or signs all have to be placed at proper parts of a product and each sign has to be easy to distinguish and recognize by a user. 


\section{Conclusion}

Overall the develop of the theory of product design in this research is based on three rules. One is a design of outward appearances according to the needs of functions and also for covering inner components. Another one is about ergonomics for operation emphasizes on body sizes and a design which is based on operation needs. The last one is based on the technology today and its manufacturing skills or needs of material properties; it's a tendency of quantity and modeling focused. All the above come with limits, they are product but people orientated. In today's society, our living environment has become focused on "people" instead of "objects". Therefore the main focus of design is humanism[6], and the style of the product should be focused on communication with "people".

Through the study in the article we can experience various product designs with mental knowledge. Can we use a mark to form a product? And being able to present a clear image of a product's spirit. Signs can guide a user through operation smoothly, and that is the goal designers all share. Hopefully the result of this research will help to clarify "the meaning of product signs" and its meaning and value, and being able to use it in designs of product forms, colors and textures. Therefore, when it comes to product design, we should use semantic signs to the best according to a user's habits and experiences, that is the goal of product design. The main focus of this study is how to use signs and symbols in product design in a proper manner.

\section{References}

1. Lin, C.H.: Design Theory and Value. Garden City Publish, Taipei (2001)

2. Marx, P., Wartburg, Elizabeth, Hsu (Translate): The Design Application of the Mass Semiotics. China Social Science Publish, Beijing (1992)

3. The semiotics theory is judging "the form" to express the significance, The semiotics theory is using the symbol to display the design significance. such an expression way belongs to the function and the spirit

4. Languish, J., Lin, S.H.: Objects and Images, Product Semantics any use?, pp. 132-133. University of Industrial Arts, Helsinki (1992)

5. Ho, C.-Y., Shih, R.: On the Relation Between Product Semantics and Semiotics, p. 6. University of Technology, Taipei (1997)

6. Kuang, C.L.: Contrasting Designing, p. 23. The Artist Publish, Taipei (1995) 